\title{
High-order LES of the flow over a simplified car model
}

\section{On the influence of the Reynolds number}

\author{
Matthieu Minguez**** — Richard Pasquetti** — Eric Serre* \\ * Laboratoire MSNM-GP, UMR CNRS 6181, Université Aix-Marseille \\ IMT La Jetée - Technopôle de Chateau-Gombert \\ 38, rue Frédéric Joliot Curie, F-13451 Marseille cedex 20 \\ \{Minguez,Eric.Serre\}@L3M.univ-mrs.fr \\ ** Laboratoire J.-A. Dieudonné, UMR CNRS 6621 \\ Université de Nice-Sophia-Antipolis, Parc Valrose \\ F-06108 Nice cedex 02 \\ Richard.Pasquetti@unice.fr
}

\begin{abstract}
We study the influence of the Reynolds number on the Ahmed body flow for the subcritical incidence $\alpha=25^{\circ}$. Large Eddy Simulations (LES) have been performed at low Reynolds number $R e=8322$, in agreement with the experiments of Spohn and Gillieron (2002), for the configuration used by Lienhart et al. (2002) at Re=768000. Our simulations, based on a spectral Chebyshev collocation-Galerkin Fourier method, have been carried out with a parallel multi-domain solver. The LES capability is implemented by a Spectral Vanishing Viscosity $(S V V)$ technique. The results globally point out that the topology of the flow is essentially determined by the body geometry.

RÉSUMÉ. Nous étudions l'influence du nombre de Reynolds sur l'écoulement autour du corps d'Ahmed pour l'incidence sous-critique $\alpha=25^{\circ}$. Des simulations des grandes échelles (LES) sont présentées à bas nombre de Reynolds Re $=8322$, conformément aux expérimentations de Spohn et Gillieron (2002), dans la configuration utilisée par Lienhart et al. (2002) à $R e=768000$. Nos simulations, utilisant une méthode d'ordre élevé de type collocation Chebyshev-Galerkin Fourier, sont réalisées à l'aide un solveur multidomaine parallélisé. La LES est introduite par une technique de viscosité spectrale évanescente (SVV). Les résultats montrent que la topologie globale de l'écoulement est essentiellement déterminée par la géométrie du corps.

KEYWORDS: large-eddy simulation, spectral methods, spectral vanishing viscosity, Ahmed body.

MOTS-CLÉS : simulation des grandes échelles, méthodes spectrales, viscosité spectrale évanescente, corps d'Ahmed.
\end{abstract}

DOI:10.3166/EJCM.18.627-646 @ 2009 Lavoisier, Paris

EJCM - 18/2009. High-performance computing, pages 627 to 646 


\section{Introduction}

The understanding and the control of flows over vehicle models is of major importance, e.g. to improve fuel consumption or resolve stability problems. The Ahmed body, firstly introduced by Ahmed et al. (1984), constitutes a simplified reference car model commonly used in the automotive industry. In spite of the relative simplicity of the geometry of this bluff body, the flow developing around appears fully turbulent and complex (strong time-dependence, recirculation zones, thin boundary layers). Then, rare are the methods and the models able to correctly describe this flow. Consequently, this geometry is subject to large academical studies and constitutes a test case for the computational fluid dynamic (CFD) models.

The pioneer experimental study, whose aim was to evaluate the influence of the rear part of the body on the aerodynamic coefficients, has pointed out a critical incidence angle $\left(\alpha=30^{\circ}\right)$ for which the topology of the flow dramatically changes. For sub-critical incidences $\left(\alpha<30^{\circ}\right)$ the flow on the slant back of the vehicle is controlled by two strong contra-rotative vortices forcing the fluid to stay attached or confining a partial recirculation on the slant. At the difference, for over-critical incidences, the two trailing vortices are no more strong enough and consequently the fluid detaches at the leading edge of the slant and only reattaches in the near wake.

Within the framework of two European benchmarks, ERCOFTAC (see Manceau and Bonnet (2000)) and DFG-CNRS program (LES for complex flows), the CFD community has found an interest in computing the flow over the Ahmed body for two characteristic slant angles, $\alpha=25^{\circ}$ and $\alpha=35^{\circ}$, at Reynolds number $R e=768000$ (where $R e$ is defined from the bulk velocity $U_{\infty}$ and the Ahmed body height $h$ ). At the moment of the $9^{t h}$ and $10^{t h}$ ERCOFTAC Workshops on Refined Turbulence Modeling, many numerical RANS-methodologies (for only one LES) have been tested and compared to the reference experimental results of Lienhart et al. (2002) with different accuracy levels. If all the methods agreed with the reference results for the over-critical angle, large divergences are reported for the sub-critical incidence in the $10^{t h}$ ERCOFTAC Workshops report of Menter (see Manceau and Bonnet, (2000)). Efforts are still made today to improve and test existent turbulence models on the Ahmed body but the last RANS attempts still failed for the incidence $\alpha=25^{\circ}$, as reported in Guilmineau (2007). In this spirit, hybrid approaches have been tested on the Ahmed body configuration as Detached-Eddy Simulation (DES), Menter and Kuntz (2003), Kapadia et al. (2003), but small differences in separation prediction (and possibly reattachment) remain. More recently, an original Lattice Boltzmann method, Fares (2006), has allowed to recover the expected topology of the mean flow despite slight discrepancies are still observed on turbulence statistics profiles in the symmetry plane. Hopefully, recent LES results, Hinterberger et al. (2004), Minguez et al. (2008) at $R e=768000$ and Krajnovic and Davidson (2004), at $R e=200000$ provided at least a global agreement with the experimental observations of Lienhart et al. (2002), which is very encouraging for the LES approach. 
The present numerical study focuses on the influence of the Reynolds number. The LES has been performed using a highly accurate spectral method, efficiently stabilized by a spectral vanishing viscosity (SVV) technique. The SVV stabilization provides LES solutions that converge to the DNS (Direct Numerical Simulation) solution of the Navier-Stokes equations when increasing the SVV threshold and moreover preserves the spectral accuracy for smooth solutions. For $\alpha=25^{\circ}$, we investigate the influence of the Reynolds number on the topology of the flow by providing results at $R e=8322$, in agreement with the experimental work of Spohn and Gillieron (1999). Beyond the qualitative observations, we provide quantitative results and comparisons with former results obtained at $R e=768000$ with the same methodology, see Minguez et al. (2008).

\section{Physical and mathematical model}

The geometry of the car model conforms with the experiments of Lienhart et al. (2002). The Ahmed body, of length $l=1044 \mathrm{~mm}$, height $h=288 \mathrm{~mm}$, width $w=$ $389 \mathrm{~mm}$ and of slant incidence $\alpha=25^{\circ}$, is placed at $d=50 \mathrm{~mm}$ from the ground, as presented in Figure 1. The fluid domain is constituted of a tunnel of rectangular section, $1370 \mathrm{~mm} \times 1000 \mathrm{~mm}$ (in the $z$-spanwise and $y$-vertical directions), spreading on 4 lengths $l$ of the bluff body, which is located at the distance $l$ from the inlet. In agreement with the experiments of Spohn and Gillieron (2002) the Reynolds number is taken equal to $R e=8322$ (inlet velocity $0.1 \mathrm{~m} / \mathrm{s} \leq U_{\infty} \leq 0.3 \mathrm{~m} / \mathrm{s}$, body height $h=81 \mathrm{~mm}$ and water kinematic viscosity $\nu \approx 10^{-6} \mathrm{~m}^{2} / \mathrm{s}$ in the experiments).

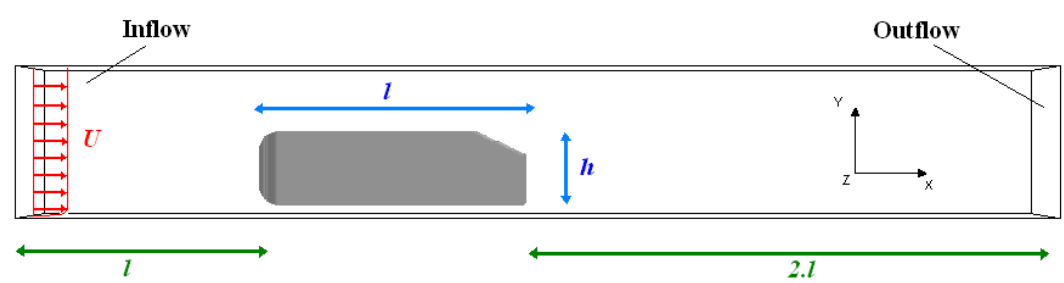

Figure 1. Schematic of the computational domain with physical characteristics

The flow is governed by the incompressible three-dimensional Navier-Stokes equations written in primitive variables, and made dimensionless using the Ahmed body height $h$ and the imposed inlet velocity $U_{\infty}$ as characteristic scales for length and velocity, respectively. Hereafter, all the quantities are thus dimensionless. The boundary and initial conditions associated to the governing equation are the following. At the initial time, the fluid is at rest. Boundary conditions are only required for the velocity: They are of no slip type at the ground $(y=0)$ and around the bluff body. The upper part of the domain $(y=3.47)$ is treated as a free slip surface, as e.g. proposed for the $10^{\text {th }}$ ERCOFTAC Workshops on Refined Turbulence Modeling 
(see Manceau and Bonnet (2000)), and periodicity is assumed in the spanwise direction. Inlet and outlet boundary conditions are more tricky. At the inlet, the velocity is constant in time and show a stiff variation to vanish at the ground. At the outlet convective boundary conditions at the mean flow velocity are applied.

\section{Numerical model}

For the paper to be self contained, this Section briefly describes the numerical method. More details may be found elsewhere, especially in Minguez et al. (2007).

\subsection{Spatial and time approximation}

The numerical method is based on a multi-domain Chebyshev-Fourier approximation. In the streamwise direction, the computational domain is decomposed in non overlapping sub-domains of different lengths depending on the flow region. The continuity of the solution at the sub-domain interfaces is ensured by using an influence matrix technique, as in Sabbah and Pasquetti (1998). In each sub-domain, a collocation Chebyshev method is used in the vertical and streamwise directions whereas a Fourier-Galerkin method is used in the spanwise periodic direction.

The Gauss-Lobatto-Chebyshev (GLC) mesh is especially adapted when boundary layers occur at the boundary of the computational domain, since GLC points accumulate at the end-points of the reference interval $(-1,1)$. Consequently, to take care of the boundary layers which develop around the bluff body, we use a mapping to accumulate grid-points at the roof of the car model in the vertical $y$-direction, see Figure 2 . In the streamwise $x$-direction we use the natural refinement of the GLC points by locating sub-domain interfaces precisely at the front and rear parts of the Ahmed body, cf. Figure 2. Moreover, a sub-domain interface also coincides with the beginning of the slant in order to correctly describe the flow at the detachment line.

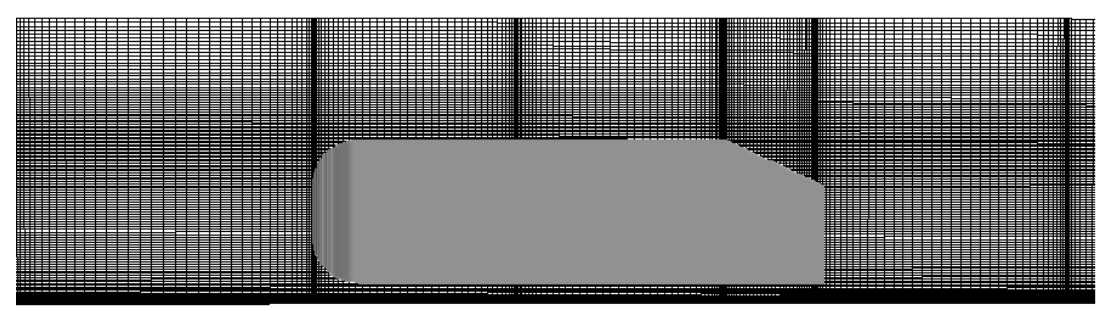

Figure 2. Mesh grid around the Ahmed body 
The discretization in time is based on a fractional step method. Globally $2^{\text {nd }}$ order accurate it makes use of the following three steps, as detailed in Cousin and Pasquetti (2004) and references herein :

- An explicit transport step, based on an Operator Integration Factor (OIF) semi Lagrangian method and a $4^{\text {th }}$ order Runge-Kutta scheme to handle the non-linear convective term.

- An implicit diffusion step, to handle the linear viscous term. Time derivatives of the velocity components are approximated at the resolution time $t_{n+1}$ with second order backward differences and the pressure is expressed at time $t_{n}$, using the so-called Goda scheme, to obtain a provisional velocity.

- A projection step, to obtain a divergence free velocity field. It is based on an unique grid " $\mathbb{P}_{N}-\mathbb{P}_{N-2}$ " approximation, so that no boundary conditions are required for the pressure. It is indeed a Darcy type problem rather than a Poisson one which is solved at this step. In our implementation, the $\mathbb{P}_{N}-\mathbb{P}_{N-2}$ approximation, where the polynomial degree for the pressure is two degrees less than for the velocity components, has been extended to the case of our multi-domain approximation : Unknown values of the pressure are considered at all the inner grid-points, including the subdomain interface points.

\subsection{Spectral vanishing viscosity methodology for LES}

In the frame of spectral approximations, which are much less diffusive than low order ones, high-Reynolds number flows are difficult to compute. The accumulation of energy in the high spatial frequency range generally leads to a divergence of the computations. As a solution, a spectral vanishing viscosity (SVV) technique is proposed as an efficient stabilization method of a highly accurate spectral approximation. It consists in modifying the Navier-Stokes equations, by introducing a viscous term acting only on the highest resolved-frequencies, to obtain a new set of equations more amenable to approximate the exact solution. Contrarily to many stabilization techniques, that generally destroy the spectral accuracy of the algorithm, see e.g. Guermond (2004), SVV possesses the property of preserving the spectral accuracy.

First introduced by Tadmor (1989) to solve non-linear hyperbolic equations, typically the Burgers equation, using standard Fourier spectral methods, the SVV method for the LES (SVV-LES) of incompressible turbulent flows has already shown its effectiveness through different works, see e.g. Karamanos and Karniadakis (2000), Kirby and Karniadakis (2002), Pasquetti (2006-a) and more recently Severac et al. (2007).

In our implementation, the SVV term is introduced in the Navier-Stokes equations through a new viscous term $\Delta_{S V V}$, so that the numerical approximation, say 
$\left(\mathbf{u}_{N}, p_{N-2}\right)$, solves (in the collocation Chebyshev-Galerkin Fourier sense) the semidiscrete system :

$$
\begin{aligned}
& \partial_{t} \mathbf{u}_{N}+\mathbf{u}_{N} \cdot \nabla \mathbf{u}_{N}=-\nabla p_{N-2}+\nu \Delta_{S V V} \mathbf{u}_{N} \\
& \nabla \cdot \mathbf{u}_{N}=0
\end{aligned}
$$

with $\nu$ for the inverse of the Reynolds number and where $\Delta_{S V V}$ is defined as :

$$
\Delta_{S V V} \equiv \nabla \cdot S_{N} \nabla
$$

and where $S_{N}$ is the diagonal operator:

$$
S_{N}=\operatorname{diag}\left\{S_{N_{i}}^{i}\right\}, S_{N_{i}}^{i}=1+\frac{\varepsilon_{N_{i}}^{i}}{\nu} Q_{N_{i}}^{i}
$$

with the subscript $i$ to denote the $i$-direction (we use here $x_{i}$ for $x, y$ and $z$ ) and where appear the amplitude coefficient and spectral viscosity operator, $\epsilon_{N}$ and $Q_{N}$ in $1 \mathrm{D}$, as introduced in the periodic case (Fourier approximation) by Tadmor (1989) and in the non-periodic case (Legendre approximation) by Maday et al. (1993). Let us recall that $\epsilon_{N}$ is usually a $O(1 / N)$ coefficient and the operator $Q_{N}$ acts on the upper part of the Fourier, Legendre or Chebyshev spectrum of the spectral approximation : With e.g. $\varphi_{k}$ for the Legendre polynomial of degree $k$, if $v=\sum_{k=0}^{\infty} \hat{v}_{k} \varphi_{k}$, then $Q_{N}(v)=\sum_{k=0}^{N} \hat{Q}_{k} \hat{v}_{k} \varphi_{k}$, with $1 \geq \hat{Q}_{k}>0$ if $k>m_{N}$ and $\hat{Q}_{k}=0$ if $k \leq m_{N}$, with e.g. $m_{N}=\sqrt{N}$. In practice we use the formula introduced by Maday et al. (1993), $\hat{Q}_{k}=\exp \left(-(k-N)^{2} /\left(k-m_{N}\right)^{2}\right)$ if $k>m_{N}$. As mentioned earlier, to refine the mesh around the bluff body a mapping is required. Since the polynomial approximation holds in the reference domain, say $\hat{\Omega}$, with the mapping $\mathbf{f}: \hat{\Omega} \rightarrow \Omega$, the operator $S_{N}$ is defined as follows :

$$
S_{N}(\nabla \mathbf{u}) \equiv S_{N}(\hat{\nabla} \hat{\mathbf{u}}) G
$$

where $G$ is the Jacobian matrix of $\mathbf{f}^{-1}$ and $\hat{\mathbf{u}}=\mathbf{u} \circ \mathbf{f}$.

The practical implementation of the operator $\Delta_{S V V}$ is based on the introduction of SVV modified differentiation matrices. From the previous definitions of $S_{N}$ and $\Delta_{S V V}$ we indeed have :

$$
\left[\Delta_{S V V} \mathbf{u}\right]_{i}=\left[\nabla \cdot S_{N}(\nabla \mathbf{u})\right]_{i}=\sum_{j} \partial_{j}\left(\tilde{\partial}_{j} \mathbf{u}_{i}\right)
$$

where $\tilde{\partial}_{j}=\left(1+\nu^{-1} \epsilon_{N_{j}} Q_{N_{j}}^{j}\right) \partial_{j}$.

The first theoretical works of Tadmor (1989) have pointed out admissible ranges for the SVV-parameters. Additionally, in the LES frame these values must correspond to a compromise between the accuracy of the SVV solution and the stability of the numerical scheme. In the present study, out of the boundary layers developing around the bluff body, the SVV parameters have been chosen isotropic independently of the spatial $(x, y, z)$ directions as $: \epsilon=1 / N$ and $m_{N}=\sqrt{N}$. 


\subsection{Obstacle modeling}

The Ahmed Body is modeled using a volume penalization method which consists in introducing an additional term canceling (approximately) the velocity field in the volume of the obstacle. The main advantage of such a technique is that complex geometries may be considered with simple meshes, so that very efficient solvers can be used. Using the standard volume penalization method, the Navier-Stokes equations are penalized as follow :

$$
\partial_{t} \mathbf{u}_{N}+\mathbf{u}_{N} \cdot \nabla \mathbf{u}_{N}=-\nabla p_{N-2}+\nu \Delta_{S V V} \mathbf{u}_{N}-C \chi(\mathbf{x}) \mathbf{u}_{N}
$$

where $C$ is a penalization constant and $\chi(\mathbf{x})$ the characteristic function of the obstacle, equal to 1 inside the bluff body and 0 elsewhere.

This approach has motivated a lot of numerical as well theoretical studies, see e.g. Angot et al. (1999) or Khadra et al. (2000). The main problem is that the penalty term may induce a stability problem, if handled explicitly, or ill conditioned systems of equations, if handled implicitly. To overcome this difficulty, the pseudo-penalization method described in Pasquetti et al. (2008) is implemented. To introduce this technique, let us restart from the Navier-Stokes system (momentum and continuity equations) and assume that the linear diffusive term is treated implicitly, whereas the nonlinear convective term is treated explicitly. Then the following semi-discrete equations must be solved at each time-step :

$$
\begin{gathered}
\nu \Delta_{S V V} \mathbf{u}_{N}^{n+1}-\frac{\alpha}{\tau} \mathbf{u}_{N}^{n+1}-\nabla p_{N-2}^{n+1}=\mathbf{f}^{n+1} \text { in } \Omega \\
\nabla \cdot \mathbf{u}_{N}^{n+1}=0
\end{gathered}
$$

where $n$ is the time index, $\tau$ the time-step and $\alpha$ a scheme dependent coefficient ( $\alpha=3 / 2$ for a second-order backward finite difference scheme). The pair $\left(\mathbf{u}_{N}^{n}, p_{N-2}^{n}\right)$ is the numerical approximation of $(u, p)$ at time $t_{n}$ and $\mathbf{f}^{n+1}$ is an easily identifiable source term, which also depends on the time scheme.

The obstacle, defined by the characteristic function $\chi$, being embedded in the computational domain $\Omega$ the pseudo-penalization method consists in solving :

$$
\begin{gathered}
\nu \Delta_{S V V} \mathbf{u}_{N}^{n+1}-\frac{\alpha}{\tau} \mathbf{u}_{N}^{n+1}-\nabla p_{N-2}^{n+1}=(1-\bar{\chi}) \mathbf{f}^{n+1} \text { in } \Omega \\
\nabla \cdot \mathbf{u}_{N}^{n+1}=0
\end{gathered}
$$

where $\bar{\chi}$ is a regularized characteristic function, in practice obtained from local average of the function $\chi$ and allowing to weaken the expected Gibbs phenomenon. However, as detailed in Minguez et al. (2008), for fully turbulent flows better results may be obtained without regularization, i.e. $\bar{\chi} \equiv \chi$, and numerical tests have shown that, due to the SVV, no Gibbs phenomenon impairs the solution. 


\subsection{Near-wall correction}

At very high Reynolds number, boundary layers developing around the bluff body become too thin to be resolved at a reasonable computational cost. Consequently, since the last decade large effort are made to develop near-wall models. Piomelli and Ballaras (2002) present a non-exhaustive listing of log-law traditionally used with success with finite volume method in some academical cases. However, in the frame of spectral methods and penalization techniques the near-wall treatment appears as an open and challenging problem.

In order to capture at the best the turbulent production phenomena localized in the near-wall region, keeping the global stability of the solution, we have relaxed in the boundary layers the threshold parameter $m_{N}$, in order to diminish the range of frequencies on which the SVV acts. This near-wall correction is introduced with a new characteristic function $\chi_{B L}(\mathbf{x})$, equal to 1 in the near wall $(\mathrm{NW})$ region and to 0 outside. The Navier-Stokes equations are consequently reformulated as :

$$
\partial_{t} \mathbf{u}_{N}+\mathbf{u}_{N} \cdot \nabla \mathbf{u}_{N}=-\nabla p_{N-2}+\nu \Delta_{S V V} \mathbf{u}_{N}-C \chi \mathbf{u}_{N}+\mathbf{f}_{B L}
$$

with $B L$ for Boundary Layer and where :

$$
\mathbf{f}_{B L}=\chi_{B L} \nu\left(\Delta_{S V V}^{B L} \mathbf{u}_{N}-\Delta_{S V V} \mathbf{u}_{N}\right) .
$$

The operator $\Delta_{S V V}^{B L}$ is defined like $\Delta_{S V V}$ but makes use of a greater value of $m_{N}$. Note that a smaller value of $\epsilon_{N}$ is also possible to modify the influence of the SVV terms, but would be more drastic. Moreover, in order to take into account the strong anisotropy of the flow in this region, the distribution of grid-points in the three directions and the stability of the computation, the operator $\Delta_{S V V}^{B L}$ is anisotropic. Thus, the values of the parameter $m_{N}$ differ, depending on the direction : $m_{N}=\left\{2 \sqrt{N_{x}}, 5 \sqrt{N_{y}}, 4 \sqrt{N_{z} / 2}\right\}$ in $(x, y, z)$ directions and again $\varepsilon_{N}=1 / N$. The corresponding spectral kernels $\hat{Q}_{k}$ of the SVV operator are shown in Figure 3, for two different values of $m_{N}$ and for $N=170$.

The $\mathbf{f}_{B L}$ force term is treated explicitly, using a second order Adams-Bashforth extrapolation consistent with the accuracy of the time discretization. It should be mentioned that this explicit treatment has never induced extra numerical stability constraint, certainly due to the local effect of the term $\mathbf{f}_{B L}$.

The resolution in the near-wall region around the bluff body and at the ground has been estimated using the wall coordinates $\left(x^{+}, y^{+}, z^{+}\right)$, which make use of the ratio $\nu / u_{\tau}$ for reference length. The friction velocity $u_{\tau}$ has been calculated from the simplified boundary layer equation (Tennekes and Lumley (1972)) :

$$
<-u^{\prime} v^{\prime}>+\nu \partial_{y} U=u_{\tau}^{2}
$$

where $y$ is the wall normal coordinate, $U=<u>$ the mean streamwise velocity, $u^{\prime}, v^{\prime}$ the fluctuations of the $x$ and $y$ components of the velocity, respectively. When 


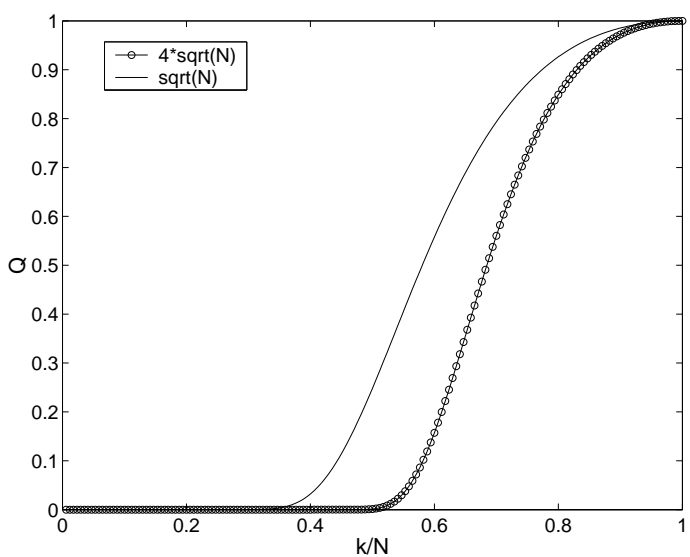

Figure 3. Spectral kernel of the one-dimensional SVV operator for different values of the threshold frequency $m_{N}, N=170$

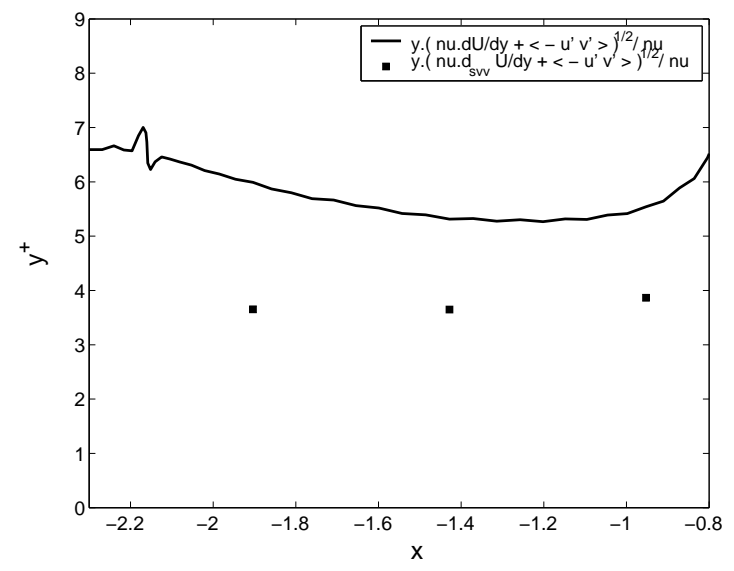

Figure 4. Resolution on the roof of the Ahmed body. Calculation of $y^{+}$along the streamwise direction in the symmetry plane $z=0$ at Re $=8322$. Here $d_{\text {svv }}$ corresponds to $\tilde{\partial}_{y}$

taking into account the SVV stabilization it is however relevant to substitute to $\partial_{y}$, in [14], the SVV modified differentiation operator $\tilde{\partial}_{y}$, as defined in Section 3.2.

The wall normal coordinate of the first grid point over the roof of the body are presented in Figure 4. From the SVV modified expression of $u_{\tau}$, one obtains $y^{+} \approx 4$, 
which is not so bad. At the ground, the value of $y^{+}$, not presented here but close to 0.2 , indicates that the boundary layer is well resolved.

\section{Computational details and performances}

The solver is parallelized using the MPI library and optimized on a NEC SX8 parallel-vectorial computer. The computational domain is decomposed in height subdomains in the $x$-streamwise direction, each of them being associated to a vectorialprocessor and discretized in $N=\left\{N_{x}, N_{y}, N_{z}\right\}$ grid-points. Three subdomains are localized around the Ahmed body, with one of them dedicated to the slant region, cf. Figure 2.

Performance tests have been carried out for four meshes : $N_{1}=\{11,61,100\}$, $N_{2}=\{21,91,160\}, N_{3}=\{31,131,240\}, N_{4}=\{41,191,340\}$. Computational costs and memory sizes are detailed in Table 1 .

Table 1. Computational performances

\begin{tabular}{c|cccc}
\hline & Preprocessing time & CPU time / iteration & Memory & Speed \\
\hline$N_{1}$ & $2.506 s$ & $1.934 \mathrm{~s}$ & $1.738 \mathrm{sB}$ & 8.332 GFlops \\
\hline$N_{2}$ & $10.065 \mathrm{~s}$ & $3.760 \mathrm{~s}$ & $3.261 \mathrm{~GB}$ & 24.692 GFlops \\
\hline$N_{3}$ & $41.169 \mathrm{~s}$ & $6.653 \mathrm{~s}$ & $7.806 \mathrm{~GB}$ & 49.307 GFlops \\
\hline$N_{4}$ & $178.618 \mathrm{~s}$ & $9.695 \mathrm{~s}$ & $19.607 \mathrm{~GB}$ & 85.429 GFlops \\
\hline
\end{tabular}

The SVV-LES results presented hereafter have been obtained with the mesh $N_{4}$, i.e. with about $21.10^{6}$ points. This mesh was the one used for our computations at $R e=768000$. The statistics have been obtained after getting a fully turbulent solution and have been then converged over the time length $T=12$, with a time step of $2.10^{-3}$ (6000 iterations). The reference time being equal to $h / U_{\infty}, T$ corresponds to $8.7 .10^{-2} s$. The CPU time was $9.695 s$ for one time-step, i.e. approximately $9,5.10^{-8} s$ per iteration and degree of freedom. Globally, the computations have required about 150 CPU hours and the fine grid calculations about 19.6 Gigabytes of memory.

\section{Results and discussion}

SVV-LES results have been obtained at $R e=8322$ and are compared both to the experimental observations of Spohn and Gillieron (1999) and to our former results at $R e=768000$ (Minguez et al. (2008)). Instantaneous and mean quantities as well as turbulence statistics are presented in this Section. For information, the experimental results of Lienhart et al. (2002) have been included in Figure 9 and 10. They compare favorably with the SVV-LES results, as detailed in Minguez et al. (2007). 


\subsection{Flow structures}

Globally, the flow structures at $R e=8322$ compare well with those found at $R e=768000$. The topology of the flow is shown by using the mean two-dimensional streamlines in the symmetry plane $z=0$ in Figure 5.

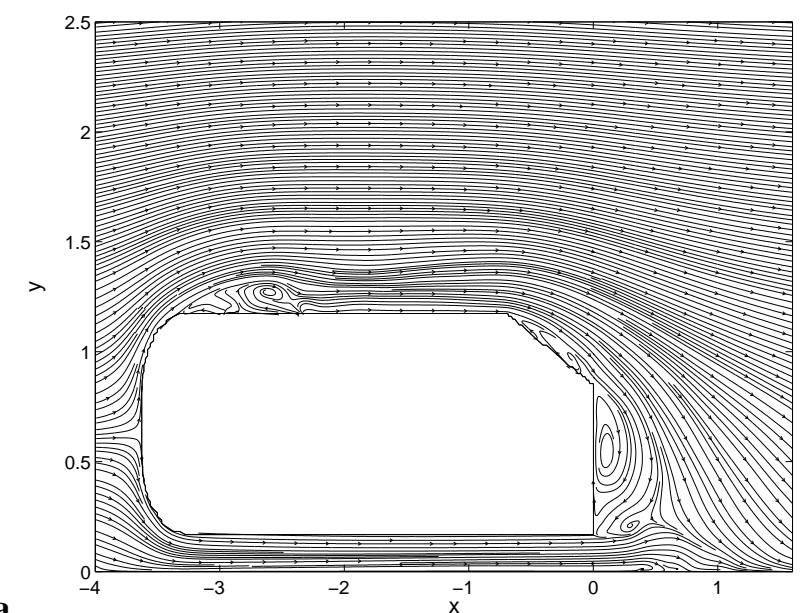

a

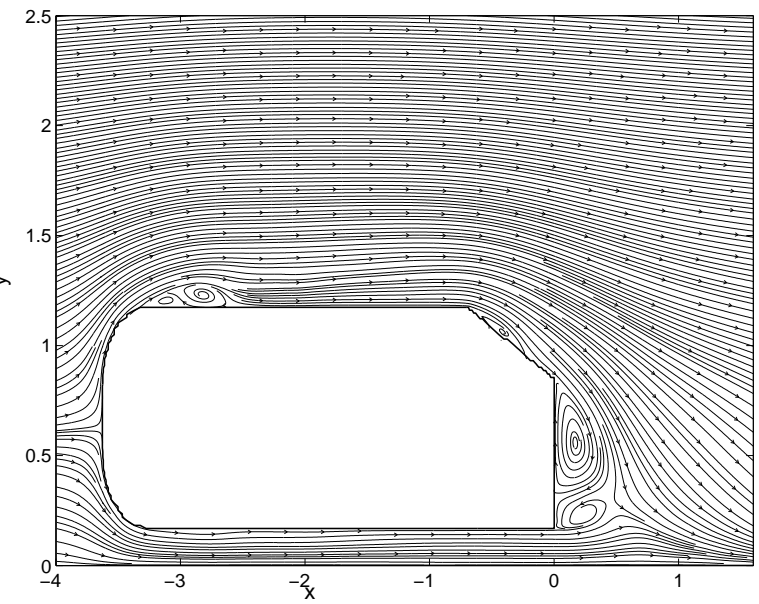

Figure 5. Mean two-dimensional streamlines over the body in the symmetry plane $z=0$ at $R e=8322$, (a), and at $R e=768000$ (Minguez et al. 2008), (b). The geometry is stretched in vertical direction to better visualize the recirculation zones

In the front part of the body, the flow separates at the beginning of the upper panel, at $x=-3.3$, and reattaches farther, at $x=-2.35$ for $R e=8322$ and at $x=-2.6$ for 
$R e=768000$. The recirculation zone induces vortex shedding, as shown in Figure 6 . Similar phenomena, not visible in Figure 5, occur on the lateral sides. In this front part of the bluff body, the laminar/turbulent transition is observable in Figure $6 \mathrm{a}$ and $6 \mathrm{~b}$. The laminar recirculation gives birth to large spanwise structures, shown by the isosurface of the pressure (Figure 6a), which then achieve a turbulent transition and form a turbulent wake, as pointed out by the iso-surface of the pressure fluctuations (Figure 6b)). All these flow recirculations have been observed experimentally by Spohn and Gillieron (1999). As expected, at the higher viscosity the recirculation zones appear longer ( $l_{r}=0.95$ instead of $l_{r}=0.7$ on the roof) and thicker than those described at $R e=768000$ in Minguez et al. (2008). Farther behind this detachment a turbulent boundary layer develops on the roof up to the sharp edge of the slant.

At the beginning of the slant, again the flow partially separates and then reattaches on the panel, but farther than at $R e=768000$. Moreover, the recirculation bubble is larger than at the higher Reynolds number, see Figure 5. In the spanwise direction, the slant-recirculation appears divided in two foci, as also observed by Spohn and Gillieron (1999) (see Figure 6a). Along the slant, as for $R e=768000$ vortical structures reminiscent of hairpin vortices develop in the shear layer of the slant-recirculation, see Figure 6. This partial slant-detachment is confined by two large counter rotating cone-like trailing vortices coming from the two edges, between the slant and the lateral surfaces of the body, and spreading farther in the wake, see Figure 7a,c,e. In addition, in the near wake helical structures roll up around these trailing vortices (see Figure 6b). With respect to the high Reynolds flow, the mean flow vortices in the wake appear both weaker and nearer of the ground, as shown by the location of the vortices center in Figure 7e,f.

For $R e=8322$, strong trailing vortices, localized at the front part and on each side, develop along the body, Figure $8 \mathrm{a}$. Such trailing vortices, not really remarkable at $R e=768000$ (Figure 7b,d,f), disappear quickly in the near wake (Figure 7a,c,e). The SVV-LES results here slightly differ from the experiments, since such trailing vortices are not reported by Spohn and Gilieron (2000) but may be discerned in the results of Lienhart et al. (2002). They have also been obtained by Krajnovic and Davidson (2001), in a LES of the flow over a shape like bus at $R e=210000$.

Finally, just behind the bluff body, two contra-rotative bubbles develop within the lower part, as shown in the symmetry plane in Figure 5. The length of this recirculation bubble, $l_{r} \approx 0.6$, is close to the one observed at $R e=768000$ (see Minguez et al. (2008), Lienhart et al. (2002)) but the lowest vortex is here of weaker intensity, certainly due to the thicker boundary layer developing under the body (report Section 5.2).

\subsection{Statistics of the turbulence}

Mean quantities are presented in this Section in the symmetry plane $z=0$, at different streamwise locations over the slant and in the wake. Moreover, additional 


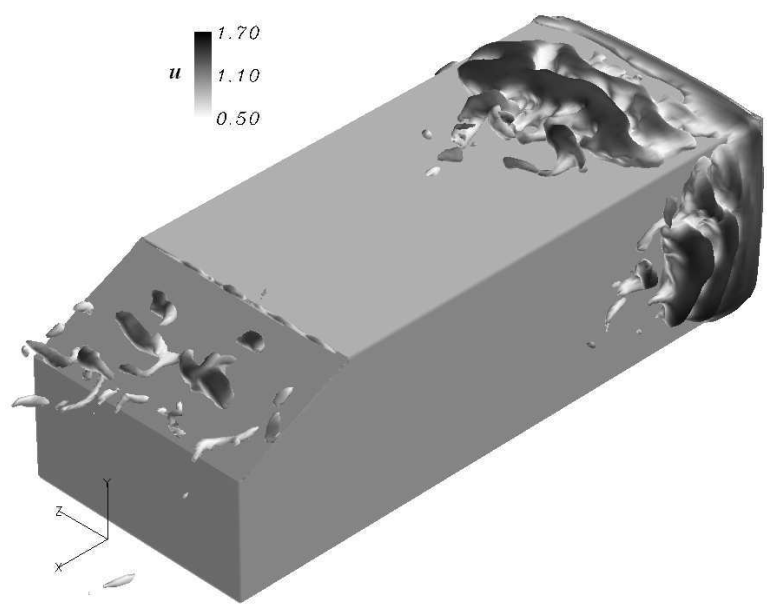

a

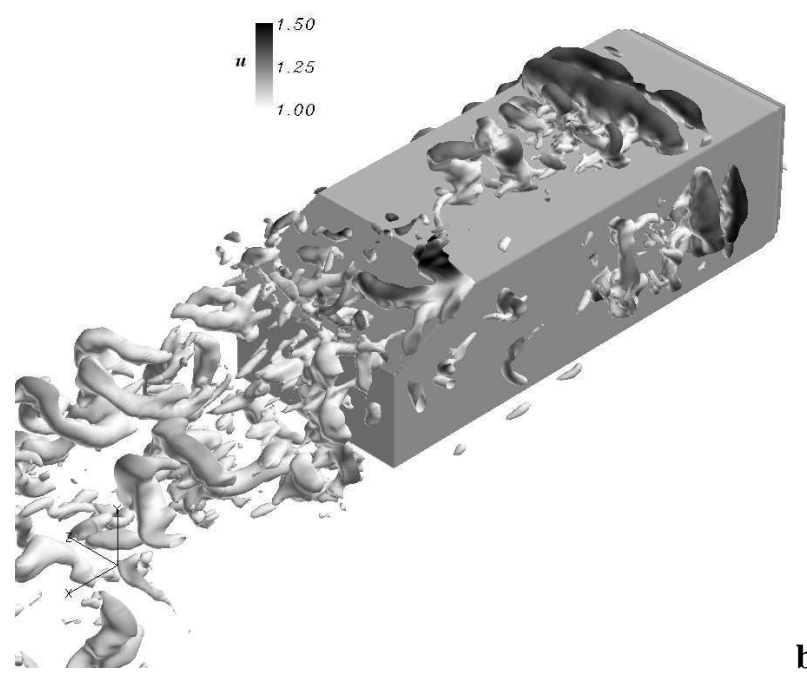

Figure 6. Iso-surface of the instantaneous pressure (a) and of the pressure fluctuations (b) colored by the instantaneous streamwise velocity $u, R e=8322$

upstream profiles of the dimensionless turbulent kinetic energy k (reference value $U_{\infty}^{2}$ ) are plotted and discussed.

At the beginning of the roof, the laminar/turbulent transition appears together with the upstream detachment, as shown by Figure 9a. The recirculation phenomenon involves a turbulent wake which thickens in the $y$-direction along the roof.This feature 
a
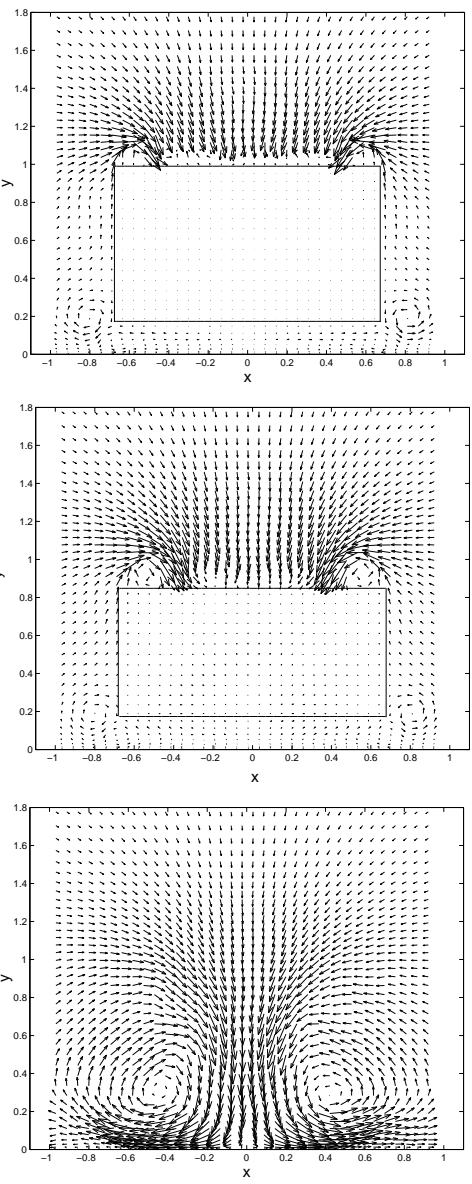
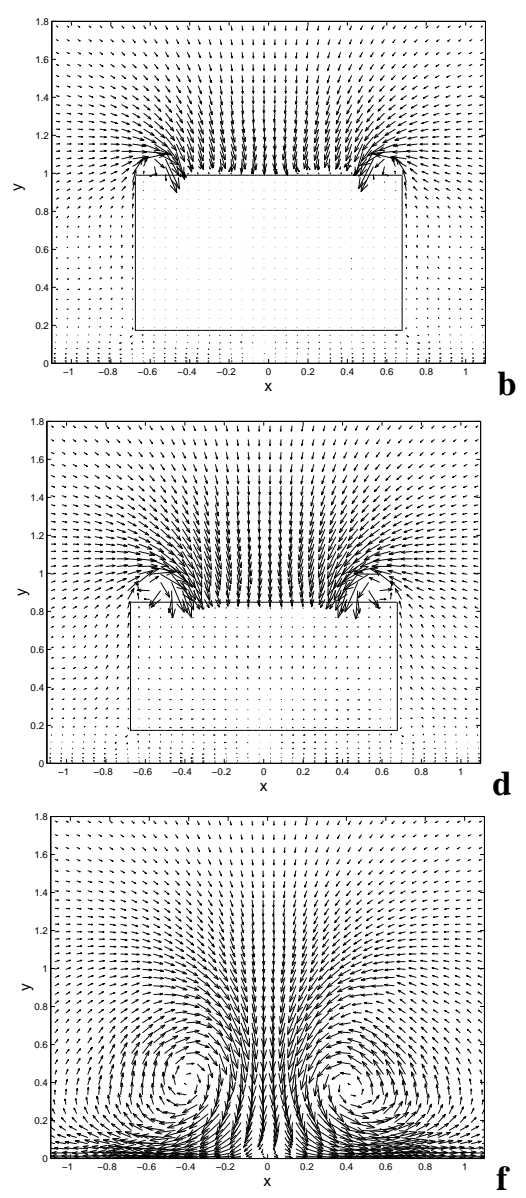

Figure 7. Velocity field in $(y, z)$ planes in the wake of the Ahmed body : SVV-LES results at $R e=8322(a, c, e)$ and SVV-LES results at $R e=768000(b, d, f)$, measured at locations $x=-0.31(a, b), x=0(b, c)$ and $x=1.34(e, f)$

seems more obvious than at high Reynolds number, certainly due to the lower mean free stream velocity (for given fluid and bluff body). In the near wall region along the roof, a thin turbulent boundary layer develops, with a dimensionless turbulent intensity weaker than at $R e=768000$ but with the same thickness, see Figure 10 .

The boundary layer over the slant thickens and partially separates due to the slant incidence. Its thickness compares to the one obtained at $R e=768000$. The fluid reattaches near the end of the slant, at $x=0.08$, i.e, farther than found by Minguez et al. (2008) at $R e=768000$, as already mentioned in Section 5.1. This larger separation moves the production of turbulence downstream to the second half part of the panel. 


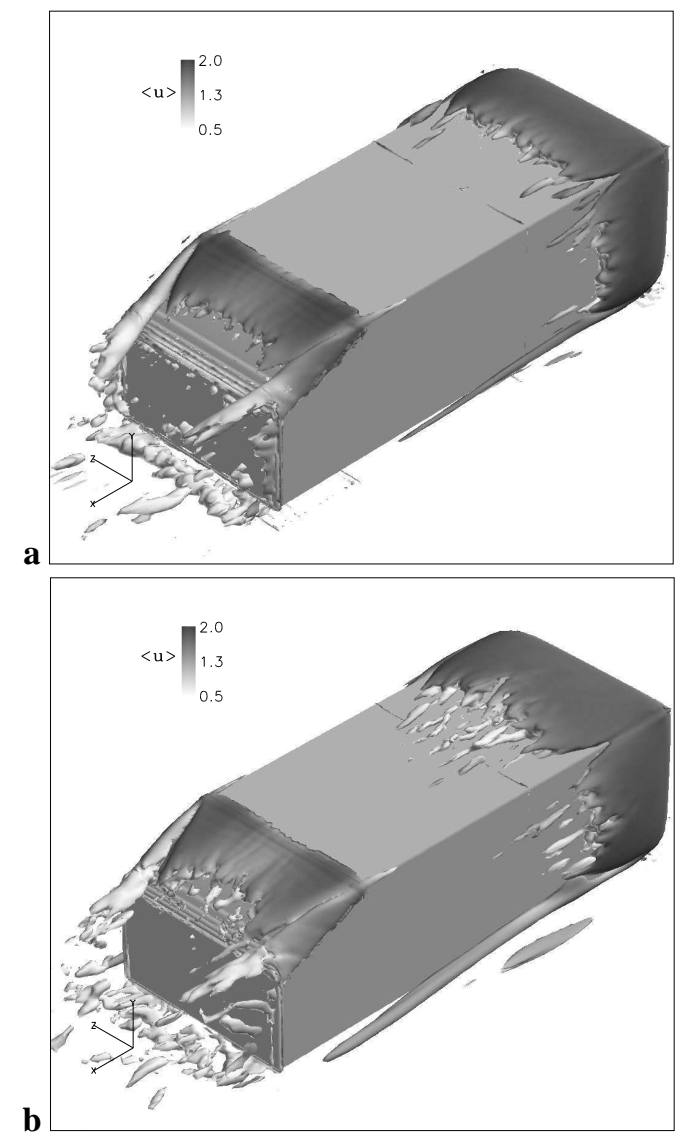

Figure 8. Iso-surface of the mean $Q$ criterium colored by the mean streamwise velocity over the Ahmed body respectively at $R e=768000,(a)$, and at $R e=8322,(b)$. $Q$ is defined as $Q=\frac{1}{2}(<\Omega>:<\Omega>-<S>:<S>)$, with $S$ the symmetrical part of $\nabla \mathbf{u}$ and $\Omega$ its anti-symmetrical part

More precisely, in the symmetry plane $z=0$ only $15 \%$ of the turbulent kinetic energy $\mathrm{k}$ is produced within the upper half part of the slant, against $45 \%$ in Lienhart et al. (2002) and 30\% in Minguez et al. (2007). These quantities have been obtained by summing numerically the turbulent kinetic energy within the near wall region over the slant, using the basic method of rectangles.

Under the Ahmed body, the laminar/turbulent transition appears at about $x \approx-1.5$ (Figure 9), whereas at $R e=768000$ the ground boundary layer which immediately turbulent and with a larger intensity. 

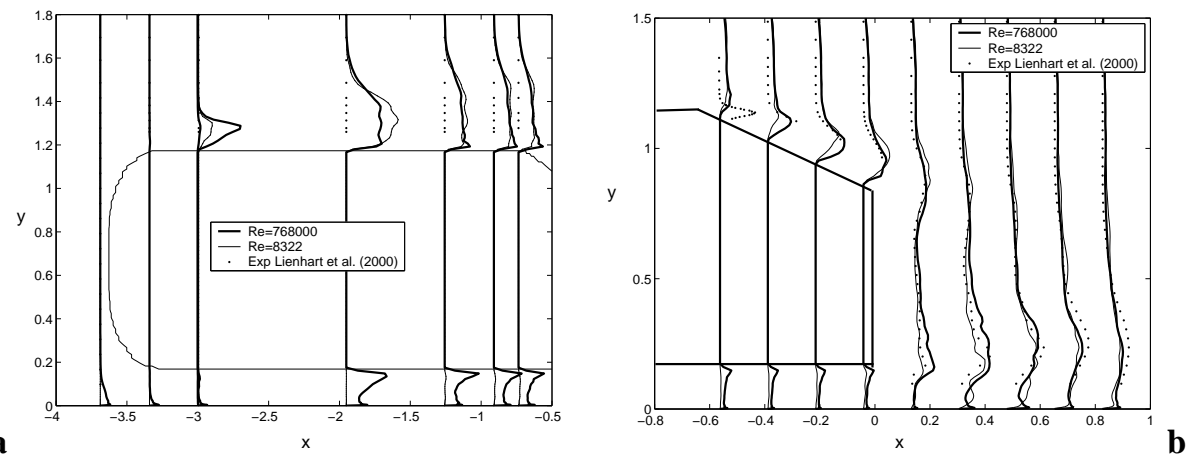

Figure 9. Profiles of turbulent kinetic energy $\mathrm{k}$ in the symmetry plane $z=0$ over the Ahmed body at Re $=8322$ on the front part (a), on the slant and in the near wake $(b)$

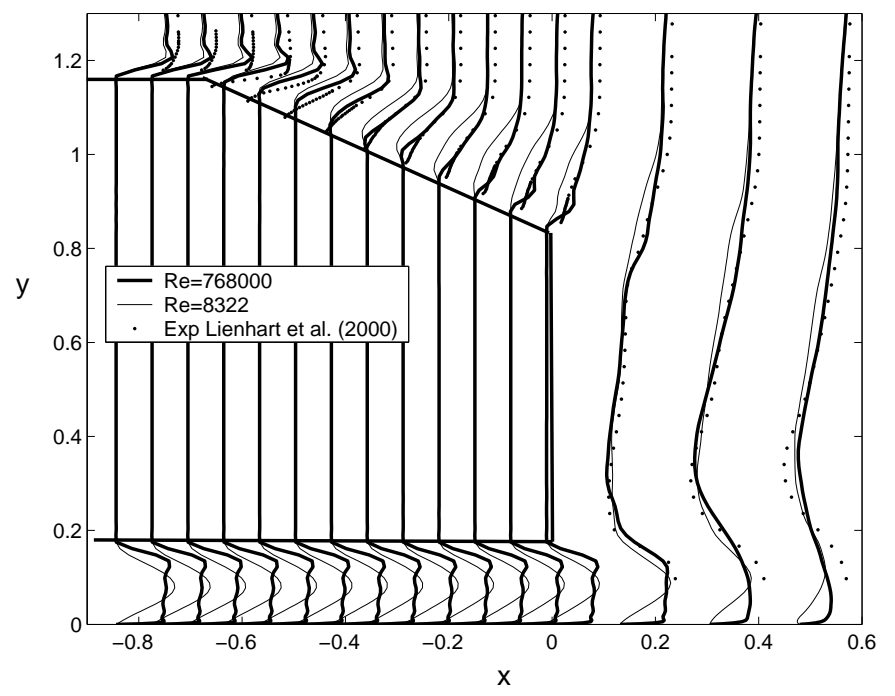

Figure 10. Profiles of mean streamwise velocity over the slant and in the wake

\subsection{Dissipation rate}

On the contrary to implicit LES or low order LES approaches, in the frame of the present high order SVV-LES one can clearly discern the contribution of the viscous and stabilization (subgrid) terms. It becomes then possible to provide a relevant computation of the dissipation rate $\varepsilon$ of the turbulent kinetic energy. 
The expression of $\varepsilon$ is deduced from the SVV stabilized Navier-Stokes equations, as detailed in Pasquetti (2006-b). Keeping the . notation for the SVV modified differentiation operators (cf Section 3.2), the dissipation rate of the turbulent kinetic energy writes as :

$$
\varepsilon=2 \nu(<S: \tilde{S}>-<S>:<\tilde{S}>) .
$$

In this expression $S$ is the usual strain rate tensor, so that :

$$
S_{i j}=\left(\partial_{i} u_{j}+\partial_{j} u_{i}\right) / 2, \quad \tilde{S}_{i j}=\left(\tilde{\partial}_{i} u_{j}+\tilde{\partial}_{j} u_{i}\right) / 2 .
$$
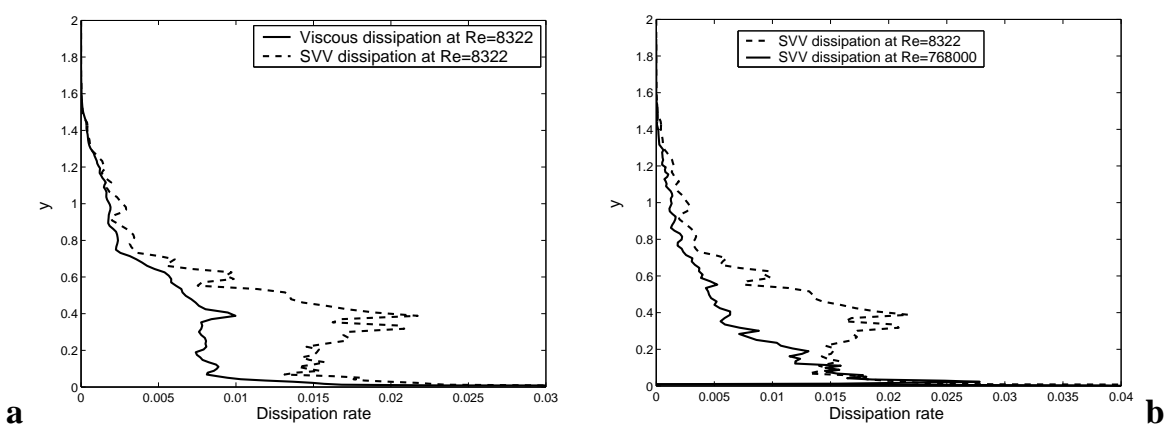

Figure 11. Dissipation rate of the turbulent kinetic energy versus the vertical direction in the symmetry plane at $x=1.51$ : Comparison of the viscous and global SVV dissipation rates at $R e=8322(a)$; Comparison of the SVV dissipation for $R e=$ 8322 and $R e=768000(b)$

The profiles of the dissipation rates in the wake (see Figure 11a) are coherent with the profiles of the turbulent kinetic energy. Indeed, the maximum of the dissipation corresponds to the maximum of $\mathrm{k}$. Moreover, the Reynolds number being lower than in Minguez et al. (2008), despite the fact that the resolutions are the same (same mesh) the balance viscous/global dissipation is much well-adjusted. In the present simulation, the SVV and viscous dissipation rates are roughly in ratio 2 at the location $x=1.51$, as shown in Figure 11a, and this tendency is recovered everywhere in the wake as over the Ahmed body. On the contrary, the SVV-dissipation at $R e=$ 768000 was in Minguez et al. (2008) two orders of magnitude larger than the viscous one. The present low Reynolds number results are then certainly more reliable than those obtained at the higher Reynolds number, which was not tackled with a finer mesh for computational cost reasons. The SVV-dissipation rates at $R e=8322$ and at $R e=768000$ are compared in Figure 11b. Of course, we present here dimensionless quantities. The reference value being equal to $U_{\infty}^{3} / h$, the dimensioned quantities may differ by a factor $(8322 / 768000)^{3}=1.3 \cdot 10^{-6}$. 


\section{Concluding remarks}

The flow over a simplified car model has been computed using a SVV-LES methodology. This investigation constitutes the first LES of Ahmed body flow at moderate Reynolds number, $R e=8322$. The results complete the ones that we obtained at the higher Reynolds number $R e=768000$, using the same mesh, i.e. with about $21.10^{6}$ grid-points.

The numerical results have well recovered the topology pointed out experimentally by Spohn and Gillieron (1999) at the same Reynolds number, particularly the slant-recirculation divided in two foci. Quantitative and qualitative comparisons, both with the LES-results of Minguez et al. (2008) and the experimental measurements of Lienhart et al. (2002) (for more details see Minguez et al. (2008)) at higher Reynolds ( $R e=768000)$, have pointed out the weak influence of the viscosity on this flow, since the topology is globally the same for the two Reynolds numbers. That confirms the observations of Krajnovic and Davidson (2004) at $R e=200000$ and shows a stronger dependence of the flow on the geometry $(\alpha)$ than on the Reynolds number $(R e)$. As expected, the low Reynold flow shows recirculation zones of larger size and weaker dimensionless turbulent intensity. Finally, the results have shown large vortical structures coming from the lower front corners of the lateral wall and spreading along the bluff body which were not reported in Spohn and Gillieron (1999).

\section{Acknowledgments}

The computations were done on the NEC-SX8 Computer of the IDRIS Computational Center (project 074055) and on the parallel computer of the SIGAMM Computational center at the OCA (Nice). The work was supported by the CNRS in the frame of the DFG-CNRS program "LES of complex flows".

\section{Bibliographie}

Ahmed S.R., Ramm G. and Faltin G., "Salient Features of the Time-Averaged Ground Vehicle Wake", SAE-Paper 840300, 1984.

Angot P., Bruneau C.H. and Fabrie P., "A penalization method to take into account obstacles in incompressible viscous flows", J. Numer. Math., vol.81, 497, 1998.

Fares E., "Unsteady flow simulation of the Ahmed reference body using a lattice Boltzmann approach", Computers \& Fluids, vol.35, n8-9, 940, 2006.

Guermond J.L., Oden J.T. and Prudhomme S., "Mathematical Perspectives on Large Eddy Simulation models for turbulent flows", J. Math. Fluid Mech., vol.6, 194, 2004.

Guilmineau E., "Computational study of flow around a simplified car body", J. Wind Eng.Ind.Aerodyn., doi :10.1016/j.jweia.2007.06.041, 2007.

Hinterberger M., Garcia-Villalba M. and Rodi W., "Large Eddy Simulation of flow around the Ahmed body", In Lecture Notes in Applied and Computational Mechanics / The Aerodynamics of Heavy Vehicles : Trucks, Buses, and Trains, McCallen, R., Browand, F., Ross (Eds.), J., Springer Verlag, 2004. 
Kapadia S, Roy S. and Wurtzler K., "Detached eddy simulation over a reference Ahmed car model", AIAA Paper 2003-0857; 2003.

Karamanos G.S. and Karniadakis G.E., "A spectral vanishing viscosity method for large-eddy simulation", J. Comput. Phys., vol.163, n²2, 2000.

Kirby R.M. and Karniadakis G.E., "Coarse resolution turbulence simulations with spectral vanishing viscosity - Large Eddy Simulation (SVV-LES)", J. Fluids Engineering, vol.124 (4), 886-891, 2002.

Khadra K., Angot P., Parneix S. and Caltagirone J.P., Fictitious domain approach for numerical modelling of Navier-Stokes equations, Int. J. Numer. Meth. Fluids, vol.34, 651-684, 2000.

Krajnovic S. and Davidson L., "Large-Eddy Simulation of the Flow Around a Ground Vehicle Body", SAE 2001 World Congress, Detroit, MI, USA, March 2001.

Krajnovic S. and Davidson L., "Flow around a simplified car", Journal of Fluids Engineering. vol.127, 907-918 et 919-928, 2004.

Lienhart H., Stoots C. and Becker S., "Flow and turbulence structures in the wake of a simplified car model (Ahmed Body)". New Results in Numerical and Experimental Fluid Mechanics, 15-17 Nov., 2002.

Maday Y., Kaber S.M.O. and Tadmor E., Legendre pseudo-spectral viscosity method for nonlinear conservation laws, SIAM J. Numer. Anal., vol.30, n², 321-342, 1993.

Manceau R. and Bonnet J.-P., "10th joint ERCOFTAC (SIG-15)/IAHR/QNET-CFD Workshop on Refined Turbulence Modelling", Poitiers, 2000.

Menter F.R. and Kuntz M., "Development and application of a zonal DES turbulence model for CFX-5",CFX Internal Rep., Otterfing, Germany, 2003.

Minguez M., Pasquetti R. and Serre E., "Spectral vanishing viscosity stabilized LES of the Ahmed body turbulent wake", ICOSAHOM 07 Congress, Beijing, 2007, and Commun. in Comput. Phys., vol.5, n²-4, 635-648, 2008.

Minguez M., Pasquetti R. and Serre E., "High-order Large-Eddy Simulation of flow over the "Ahmed body" car model", Phys. of Fluids, vol. 20, n9, 095101-095101-17, 2008.

Pasquetti R. (a), "Spectral vanishing viscosity method for large-eddy simulation of turbulent flows", ICOSAHOM 04 Congress, Brown university, 2004, and J. Sci. Comp., vol.27 (1-3), 365,2006

Pasquetti R. (b), "Spectral vanishing viscosity method for high-order LES : Computation of the dissipation rates", ECCOMAS CFD congress, P. Wesseling, J. Onate, J. Périaux (Eds), Delft The Netherland 2006.

Pasquetti R., Bwemba R. and Cousin L., "A pseudo-penalization method for high Reynolds number unsteady flows", Applied Numerical Mathematics, vol.58 (7), 946-954, 2008.

Piomelli U. and Balaras E., "Wall-layer models for large-eddy simulations", Annu. Rev. Fluid Mech., 34, 349-374, 2002.

Sabbah C. and Pasquetti R., "A divergence-free multidomain spectral solver of the NavierStokes equations in geometries of high aspect ratio", Comput. Phys., vol.139, 359, 1998.

Severac E., Poncet S., Serre E. and Chauve M.P., "Large eddy simulation and measurements of turbulent enclosed rotor-stator flows", Phys. Fluids vol.19, 085113, 2007.

Spohn A. and Gillieron P., "Flow separations generated by a simplified geometry of an automotive vehicle", In Proc. of IUATM symp on unsteady separated flows, Toulouse, 2002. 
Tadmor E., "Convergence of spectral methods for non linear conservation laws", SIAM J. Numer. Anal., vol. 26(1),30, 1989.

Tennekes H. and Lumley J., "A first course in Turbulence", The MIT Press, 1972.

Received : 17 April 2008 Accepted : 18 June 2008 